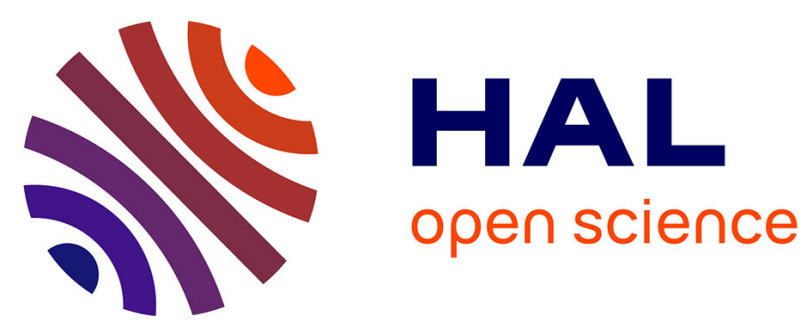

\title{
RNTI Aggregation for multi-users multiplexing radio voice transmission for enhancing voice capacity over LTE in PMR context
}

Hang Nguyen, Cuong Nguyen Manh, Eric Georgeaux, Philippe Mège, Laurent Martinod

\section{To cite this version:}

Hang Nguyen, Cuong Nguyen Manh, Eric Georgeaux, Philippe Mège, Laurent Martinod. RNTI Aggregation for multi-users multiplexing radio voice transmission for enhancing voice capacity over LTE in PMR context. VTC Spring 2015: 81st Vehicular Technology Conference, May 2015, Glasgow, United Kingdom. pp.1 - 5, 10.1109/VTCSpring.2015.7146062 . hal-01262417

\section{HAL Id: hal-01262417 https://hal.science/hal-01262417}

Submitted on 26 Jan 2016

HAL is a multi-disciplinary open access archive for the deposit and dissemination of scientific research documents, whether they are published or not. The documents may come from teaching and research institutions in France or abroad, or from public or private research centers.
L'archive ouverte pluridisciplinaire HAL, est destinée au dépôt et à la diffusion de documents scientifiques de niveau recherche, publiés ou non, émanant des établissements d'enseignement et de recherche français ou étrangers, des laboratoires publics ou privés. 


\title{
RNTI Aggregation for Multi-users Multiplexing Radio Voice Transmission for Enhancing Voice Capacity over LTE in PMR Context
}

\author{
Manh-Cuong Nguyen and Hang Nguyen ${ }^{1} \quad$ Eric Georgeaux, Philippe Mege and Laurent Martinod ${ }^{2}$
}

\begin{abstract}
Voice capacity over Long Term Evolution (LTE), despite the fact that LTE supports high-speed data transmission, is still low due to the large data and control overhead. This is one of the primary obstacles to apply LTE to Professional Mobile Radio (PMR) system because the voice capacity is one of the main requirements of the public safety network. To overcome this issue, there are several studies aiming at reducing one of the two overheads in LTE, data or control. However, in most cases the voice capacity gain can only be improved by both reducing data and control overhead. This is because these two factors are strongly related in carrying out the resource allocation. In [1], we proposed a Multi-users Multiplexing Radio Voice Transmission method. This method clusters voice packets from different users into one same LTE packet in the downlink transmission for reducing the data overhead caused by the difference between LTE packet size and the PMR voice payload. This paper presents a new Radio Network Temporary Identifier (RNTI) aggregation method to solve the control overhead issue for the multiplexing scheme of Voice over LTE (VoLTE) in PMR context. Our method uses Physical Downlink Control Channel (PDCCH) channel with high format, created by the aggregation of PDCCH channels with low format, to transmit several RNTIs of different User Equipments (UEs) in a same multiplexing group. The number of RNTIs in one PDCCH is calculated to ensure that there is no increase of Bit Error Rate (BER) for receiving PDCCH channel. The results show that the RNTI aggregation method can increase the control capacity of the multiplexing scheme up to $170 \%$. The combination of Multi-users Multiplexing Radio Voice Transmission method and RNTI aggregation method allows reducing both data and control overhead of VoLTE in the PMR context.
\end{abstract}

Index Terms-Aggregation, RNTI, scheduling, LTE, PMR

\section{INTRODUCTION}

The Professional Mobile Radio (PMR) network, which is used for public safety operation, still bases on the second generation $(2 \mathrm{G})[2]$. To satisfy the user demands in the future, PMR has to evolve to the broadband solutions [3]. In the current broadband technologies, Long Term Evolution (LTE) standard, developed by the 3GPP (3rd Generation Partnership Project), is considered one of the potential candidates for the next generation of PMR. However, LTE is not yet optimal for

\footnotetext{
${ }^{1}$ Institut Mines-Telecom, Telecom SudParis Samovar Laboratory, UMR 5157, CNRS, Evry, France \{manh_cuong.nguyen, hang.nguyen\}@it-sudparis.eu

${ }^{2}$ Department of Security and Communication Solutions CASSIDIAN (an EADS Company), Elancourt, France \{eric.georgeaux, philippe.mege, laurent.martinod\}@ cassidian.com
}

This work was accomplished in the framework of the collaborative project SOAPS.2, labeled by the Systematic PARIS-REGION Cluster low bit rate voice communication in PMR context because of the data overhead and control overhead issues.

In PMR context, the size of voice payload is typically small, however, LTE core network is purely packet switched and it has to add Internet Protocol (IP), User Datagram Protocol (UDP), The Real-time Transport Protocol (RTP), Packet Data Convergence Protocol (DPCP), Radio Link Control (RLC), Media Access Control (MAC) headers for the data transmission. In addition, in LTE, one pair of Physical Resource Blocks (PRBs) is the smallest User Assignment Unit. The smallest LTE packet size is still too large in case that low bit rate voice communication is transmitted in high Modulation and Coding Scheme (MCS) so that LTE has to add the padding information to fill the gap in the LTE packet size. These things cause the data overhead when LTE network transmit low bit rate voice communication. Numerous studies have attempted to reduce the data overhead (e.g. [4], [5], [6]).

In LTE [7] for the transmission of Downlink (DL) and Uplink (UL) transport channels, certain associated control signaling have to be used. LTE uses Physical Downlink Control Channel (PDCCH) to carry all allocation information for both downlink and uplink shared channels. PDCCH can only use first one to three Orthogonal Frequency-Division Multiple (OFDM) symbols in each subframe to carry Downlink Control Information (DCI). These symbols are organized in Resource Elements (RE), Resource Element Group and Control Channel Element (CCE). One RE corresponds to one OFDM symbol in time domain and one subcarrier in frequency domain. One REG consists of four consecutive REs or four REs separated by one Reference Signal. One CCE comprises nine REGs. To build the PDCCH, LTE uses a number of consecutive CCEs called CCE aggregation level. The CCE aggregation level can be one, two, four or eight. The aggregation level depends on the DCI size and the effective coding rate. There are four PDCCH formats (PDCCH format $0, \mathrm{PDCCH}$ format 1, PDCCH format 2, PDCCH format 3) that correspond to four aggregation levels. In LTE, the base scheduler is Fully Dynamic (FD) scheduler. In the FD scheduler, each data packet needs to associate with a L1 control signaling (a PDCCH channel). As the voice packet is small so that the number of supported data packets in one TTI (Transmission Time Interval) is increased. Consequently, the number of required control channels is significant improvement. However, the number of PDCCH is limited because the PDCCHs can only use one to three OFDM symbols in each subframe. This can 
in-turn limit the number of simultaneous voice calls. This is defined as control overhead in LTE. To solve the control overhead of FD scheduling, Semi-persistent scheduling (SPS) [8] proposed to remain certain information (Resource block assignments, Modulation and Coding Scheme...) for a preconfigured period. During this period, if the link condition change, SPS scheduler will send a new PDCCH. In group scheduling [9], the mobile stations are clustered into group and the resource allocations are scheduled for each group instead of scheduling for each mobile station.

However, state-of-the-art methods expose many difficulties to obtain the improvement of the Voice over LTE (VoLTE) capacity. The solutions for reducing data overhead (e.g. [4], [5], [6]) do not take into account the signal overhead whenever evaluating the system performance. Imagine a scenario in which we can reduce the data overhead for payload allocation but there is no more control signal for allocating others UEs. In this scenario, we cannot increase the voice capacity. On the other hand, the solutions for control overhead such as group scheduling, semi-persistent scheduling do not examine the data overhead. These methods can economize the control signaling whereas in several cases, these methods are unable to reduce the data overhead. Consecutively, it becomes a bottleneck of the voice capacity. Some other mechanism such as TTI-bundling [10] and packet bundling [11] can reduce both overheads while they increase significantly the delay. Hence, they influence the quality of service of voice over LTE.

Therefore, in [1], we proposed a Multi-users Multiplexing Radio Voice Transmission method. This method clusters voice packets from different users into one same LTE packet in the downlink transmission for reducing the data overhead caused by the difference between LTE packet size and the PMR voice payload. In addition, the Multi-users Multiplexing Radio Voice Transmission method is used simultaneously with a new RNTI aggregation method. The RNTI aggregation method is used to solve the control overhead issue for the multiplexing scheme of VoLTE in PMR context. This method uses PDCCH channel with high format, created by the aggregation of PDCCH channels with low format, to transmit several RNTIs of different UEs in a same multiplexing group. This method allows increasing significantly the control capacity of the multiplexing scheme, but does not affect the Bit Error Rate (BER) in the transmission of PDCCH channel. The details of the RNTI aggregation method is presented in section II. The performance of this method is evaluated in section III. Section IV will give the conclusion and some perspectives.

\section{RNTI AGgREgATION FOR THE MUlTI-USER MULTIPLEXING VOICE RADIO TRANSMISSION METHOD}

A. Control overhead in the Multi-users Multiplexing Radio Voice Transmission for Enhancing Voice Capacity

Figure 1 shows the control overhead issue in the Multiusers Multiplexing Radio Voice Transmission method. The control overhead issue in the Multi-user multiplexing Voice radio transmission method is similar to that in the standard LTE. The control overhead occurs when there is not enough

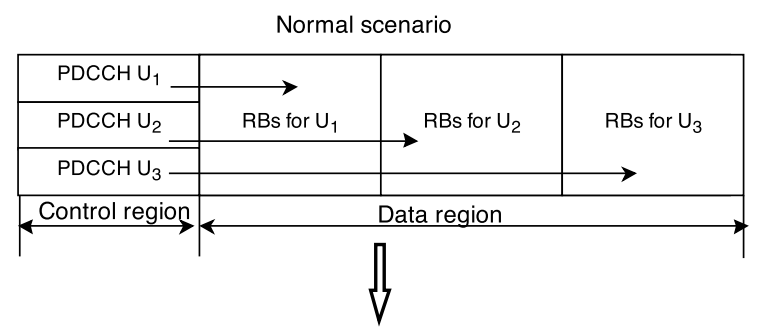

Control overhead scenario in the standard LTE

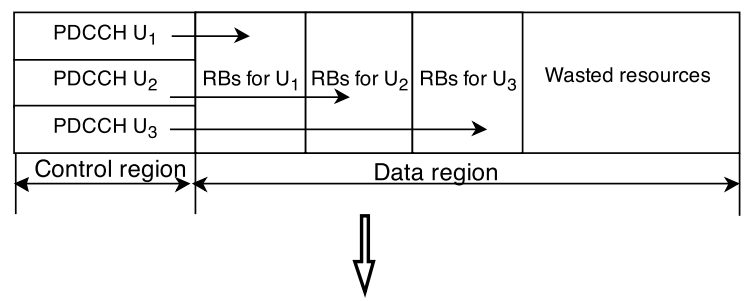

Control overhead in the Multiplexing scheme

\begin{tabular}{|c|c|c|}
\hline $\mathrm{PDCCH}_{1}$ & $\longrightarrow$ & \\
\hline $\mathrm{PDCCHU}_{2}$ & RBs for $U_{1} \& U_{2} \& U_{3}$ & Wasted resources \\
\hline $\mathrm{PDCCH} \mathrm{U}_{3}$ & & \\
\hline Control region & \multicolumn{2}{|c|}{ Data region } \\
\hline
\end{tabular}

Fig. 1. Control overhead issue of VoLTE in PMR context in the multiplexing scheme

PDCCH channels for the resource allocation. However, in the multiplexing scheme, UEs in a same group have the same allocation information (same DCI value) so that an adequate method for this situation needs to be considered.

\section{B. General idea}

Figure 2 shows the general idea of our method. Assume that there are $n$ UEs that will be scheduled in one TTI by our multiplexing algorithm. In our proposed method, instead of transmitting $n$ different PDCCHs, which contain only a same DCI information and a different RNTI for each UE in the group, we can transmit $m$ new aggregation PDCCH channels. This is done by a negligible changing in the structure of PDCCH. One PDCCH channel now can transmit one DCI value and one or several RNTIs. The number of RNTIs $\left(\left(\delta_{i}\right)\right)$ transmitted in one PDCCH depends on the DCI size, the PDCCH format that UEs required and the PDCCH which will be used for transmitting the aggregation RNTIs.

The major issues to be considered is how to determine the number of RNTIs $\left(\delta_{i}\right)$ that can be put in one PDCCH and how to ensure that the new method do not increase the bit error rate of PDCCH. In subsection II-C, we will describe our proposed mechanism for determining the RNTI aggregation level.

\section{Proposed RNTI aggregation size determination function and definition of the control capacity gain}

As LTE supports different PDCCH formats (depends on the DCI size and the code rate) and to ensure the Bit Error Rate (BER) of PDCCH, we propose of using PDCCH channels with 


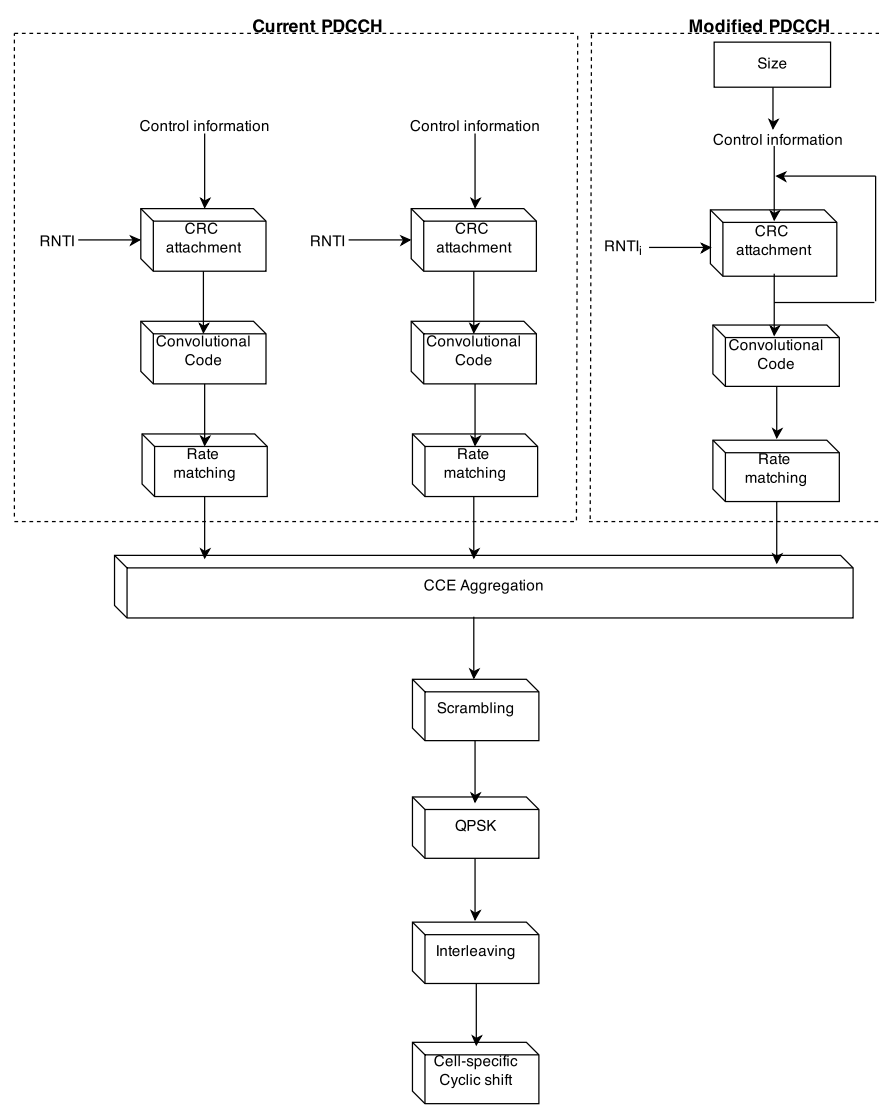

Fig. 2. RNTI aggregation general idea

high format, created by the aggregation of PDCCH channels with low format, to transmit more than one RNTIs in one PDCCH. This allows increasing the control capacity, but do not affect the BER in the transmission of PDCCH channel.

Assume that we have $n_{i}$ PDCCH channels with format $i$, in the Fully Dynamic Scheduling in LTE, with $n_{i} \mathrm{PDCCH}$ channels with format $i$ we can only transmit the allocation information (DCI) for $n_{i}$ UEs with $n_{i}$ different RNTI values. Therefore, total of UEs that can be scheduled in one TTI with the FD scheduler is:

$$
n_{\text {totalFD }}=\sum_{i=0}^{3} n_{i}
$$

In the proposed method, we aggregate $n_{i}$ PDCCH channels with format i to create $n_{i j}$ PDCCH channels with format $j$ with $j \in\{i . .3\}$. Assume that one PDCCH channel with format $j$ can transmit $\delta_{i j}$ RNTIs of UEs that require PDCCH format $i$. So in our method the total of UEs that can be total scheduled in one TTI can be estimated by equation 2 :

$$
n_{\text {totalof ProposedMethod }}=\sum_{i=0}^{3} \sum_{j=i}^{3} n_{i j} * \delta i j
$$

The control capacity gain is given by:

$$
\text { gain }=\frac{n_{\text {totalof ProposedMethod }}-n_{\text {totalFD }}}{n_{\text {totalFD }}} * 100 \%
$$

To ensure that the proposed method does not affect the BER in the transmission, the number of RNTI of UEs with PDCCH level $i$ that can be put in one PDCCH with format $j$ denoted by $\delta i j$ can be calculated by formula 7 .

Assume that in standard LTE, FD scheduler use one PDCCH channel with format $i$ with size $s(i)$ to transmit DCI with size DCIsize at code rate $r$. So we have:

$$
(\text { DCIsize }+16) * r=s(i)
$$

Where: 16 is the size of CRC xor RNTI.

In our case, we aggregate PDCCH channels of lower format $i$ to create PDCCH with higher format $j$ to transmit DCI with size DCI size at code rate $r$ of $\delta i j$ RNTI values so we have:

$$
(D C I s i z e+16 * \delta i j) * r=s(j)
$$

From Formula 4 and Formula 5 we have:

$$
\frac{D C I \text { size }+16 * \delta i j}{D C I s i z e+16}=\frac{s_{j}}{s_{i}}
$$

So:

$$
\frac{\text { DCIsize }+16 * \delta i j}{\text { DCIsize }+16}=2^{j-i}
$$

In the standard LTE, the choice of aggregation level depends on the DCI size and the radio condition. In our case, PDCCH with high format is used not only to support multiple DCI formats and to accommodate the radio condition, but also is used to transmit several RNTIs in one PDCCH. In order to maximize the control capacity, the aggregation levels will be created in high to low order priority. This is suitable for the multiplexing scheme because in the multiplexing scheme, the number of multiplexed voice packets in one LTE packet is higher in case that quality of channels are good and vice versa. At the $i^{t h}$ TTI, assume that we use $n$ CCEs to transmit $n_{i}$ PDCCH of format $i$. To increase the number of RNTIs that can be transmitted in one $\mathrm{PDCCH}$, in our proposed method, we try to create more PDCCH in high format to transmit RNTIs of UEs that require low format PDCCHs.

$$
n_{i j}= \begin{cases}\left\lfloor\frac{n_{i}}{2^{j-i}}\right\rfloor & j=3 \\ \left\lfloor\frac{n_{i}-\sum_{k=j+1}^{3} n_{i k} * 2^{k}}{2^{j-i}}\right\rfloor & i \leq j<3\end{cases}
$$

Where

- $n_{i j}$ is number of PDCCH format $j$ that is created by the aggregation of PDCCH format $i$

- $n_{i}$ is number of PDCCH format $i$

\section{CCE indexes for RNTI aggregation}

LTE can define a default value A_RNTI (Aggregation RNTI) to calculate the CCE index of PDCCHs having more than one RNTIs. The PDCCH created by RNTIs aggregation $($ dij > 1) will be considered a PDCCH of UE having A_RNTI. In standard LTE, UE will find CCE indexes on 2 areas : common search space $(\mathrm{RNTI}=0)$ and the specific search space (C_RNTI) [12]. In our proposed method, UE 


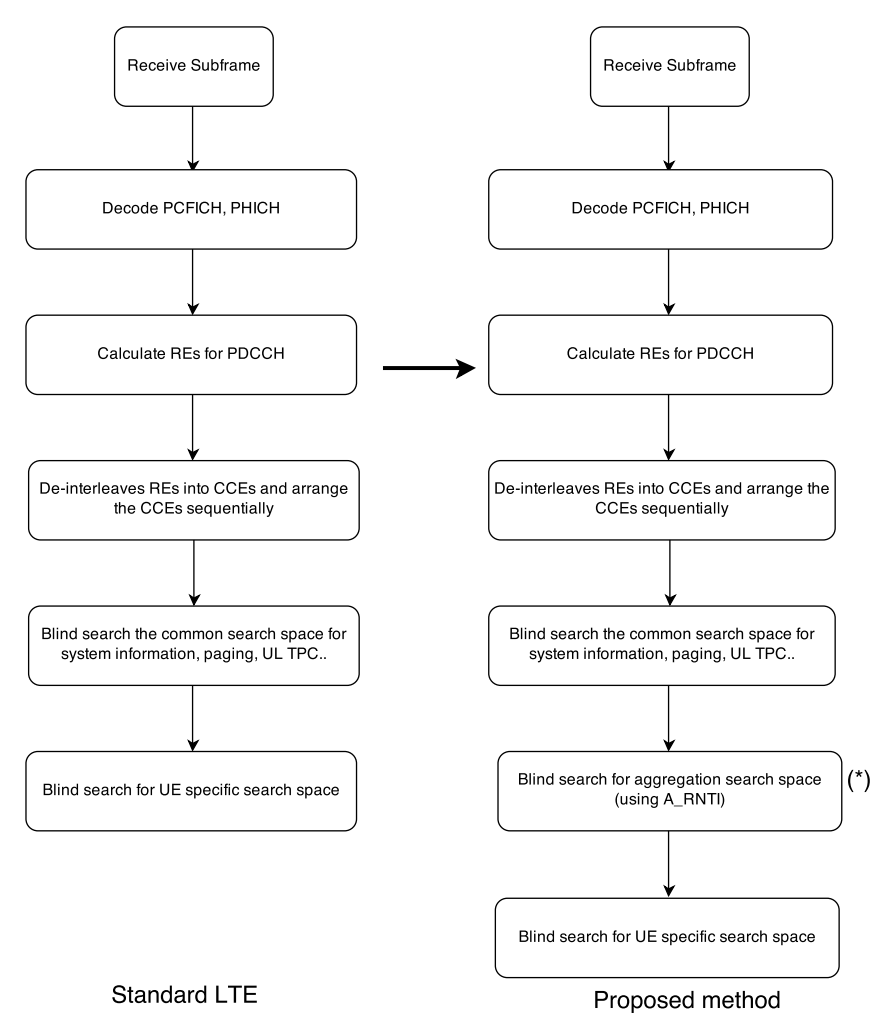

Fig. 3. PDCCH blind detection procedure of the proposed method

will search on 3 areas: common search space $(\mathrm{RNTI}=0)$, the aggregation search space (A_RNTI) and the specific search space (C_RNTI) (see figure 3).

To find the allocation in the aggregation search space, UES will first calculate the indexes CCEs by using the A_RNTI and apply the formulas in spec 36.213 [13] as:

$$
L\left(\left(Y_{k}+m^{\prime}\right) \bmod \left(\left\lfloor N_{C C E} / L\right\rfloor\right)\right)+i
$$

Where

- $L$ : Aggregation level, $\mathrm{L} \in\{1,2,4,8\}$

- $A=39827$

- $D=65537$

- $k$ : Subframe number

- $m^{\prime}=0$ to (Number of PDCCH candidates -1 )

$$
Y_{k}=\left(A . Y_{k-1}\right) \bmod D
$$

Where

- $L$ : aggregation level

- $i=0$ to (Aggregation Level - 1)

- $N_{C C E}$ : Number of CCE's available for PDCCH

- if $k=0, Y_{k-1}=A_{-} R N T I$

For each CCE index in the aggregation search space, UE will calculate all the available aggregation size values corresponding to the size of each DCI format by using the formula 7. For each aggregation size value, UEs will use its RNTI value to compare with RNTI values located in PDCCH to find out if there is the corresponding RNTI.

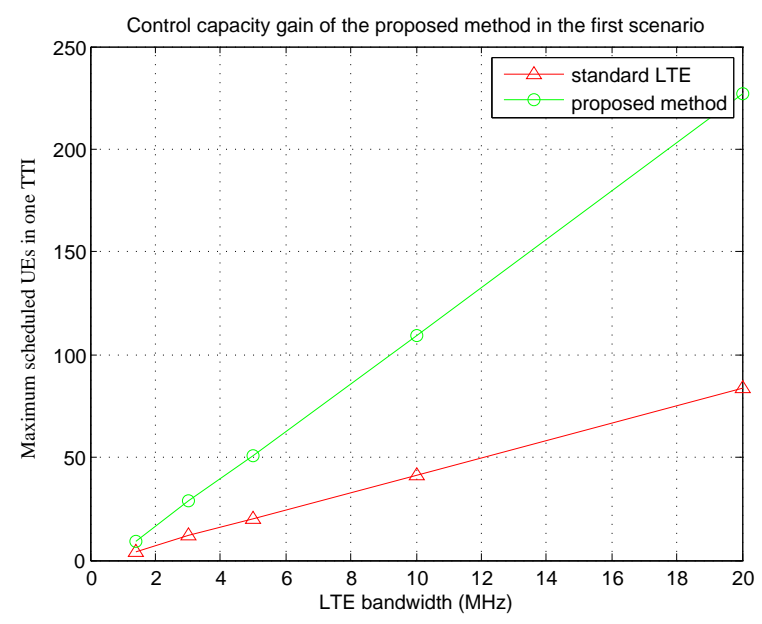

Fig. 4. Control capacity gain of the proposed method for the first scenario

\section{PERFORMANCE EVALUATION}

\section{A. Simulation Parameters}

In this section we will present simulation results of our proposed method. Two scenarios are used for our simulation. In the first scenario, we assume that all UEs use CCE with aggregation level 1 (PDCCH format 0 ) for transmission of downlink allocation information (ideal scenario). This scenario is used to estimate the maximal control capacity of the system. In the second scenario we use the model of [14]. In this model, the probability that UEs chose CCE aggregation level $i$ is $p_{i}$. With $p_{1}=0.35, p_{2}=0.25, p_{3}=0.30, p_{4}=0.1$. This model is used to estimate the control capacity gain in a more reality case. The other parameters are presented in table I.

TABLE I

SIMULATION PARAMETERS

\begin{tabular}{|l|l|}
\hline Parameter & Value \\
\hline Bandwidth & $1.4 \mathrm{MHz}, 3 \mathrm{MHz}, 5 \mathrm{MHz}, 10 \mathrm{MHz}, 20 \mathrm{MHz}$ \\
\hline Symbols for PDCCH & 3 symbols \\
\hline $\mathrm{Ng}$ & 1 \\
\hline Cyclic Prefix & Normal \\
\hline Number of antenna & 2 \\
\hline
\end{tabular}

\section{B. Simulation Results}

The maximal number of UEs that can be scheduled in one TTI between the Dynamic Scheduling and our proposed method for the first scenario are compared in Table II and Figure 4. It can be seen from the data in Table II that the proposed method reported a significant increase in the maximal number of UEs that can be scheduled in one TTI in comparison with the FD scheduling. In addition, it is apparent that the efficiency of the proposed method increases along with the increase of LTE bandwidths. In the best case, the proposed method can increase the maximal number of UEs that can be scheduled in one TTI from 84 UEs if using FD in LTE to 227 UEs (at $20 \mathrm{MHz}$ ) (170.23\%). This shows the potential of the 
TABLE II

CONTROL CAPACITY GAIN OF THE PROPOSED METHOD FOR $1 \mathrm{~ms}$ FOR THE FIRST SCENARIO

\begin{tabular}{|l|l|l|l|l|l|}
\hline Bandwidth & $1.4 \mathrm{MHz}$ & $3 \mathrm{MHz}$ & $5 \mathrm{MHz}$ & $10 \mathrm{MHz}$ & $20 \mathrm{MHz}$ \\
\hline Maximum number of CCEs & 4 & 12 & 20 & 41 & 84 \\
\hline$N_{\text {totalFD }}$ of the Fully Dynamic Scheduling & 4 & 12 & 20 & 41 & 84 \\
\hline$N_{\text {totalof ProposedMethod } \text { of the proposed method }}$ & 9 & 29 & 51 & 109 & 227 \\
\hline Control capacity gain & $125 \%$ & $141.66 \%$ & $155 \%$ & $165.8 \%$ & $170.23 \%$ \\
\hline
\end{tabular}

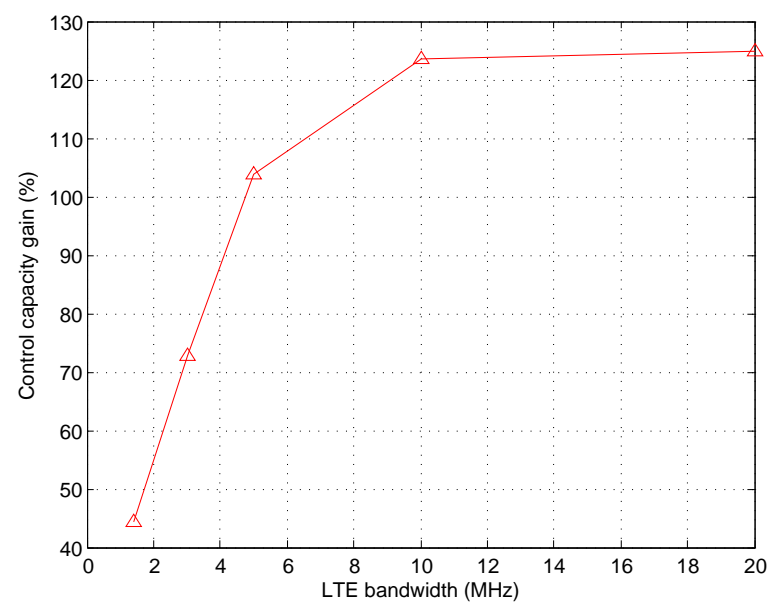

Fig. 5. Control capacity gain of the proposed method for the second scenario

method with larger LTE bandwidth. In LTE-Advanced from Release 10, there is a set of new features. One of these features is carrier aggregation. LTE-Advanced allows the aggregation of carriers to create very large bandwidths (up to $100 \mathrm{MHz}$ ). Therefore, in the case that LTE-Advanced is used for PMR context, the performance of our method will be even more interesting. The control capacity gain of the proposed method for the second scenario is shown in Figure 5. In case that the UEs are dispersed in the cell (scenario 2), the control capacity gain of the proposed method can also rise up to $124.79 \%$ (at $20 \mathrm{MHz}$ ). From the results of scenario 1 and 2, we found that our method gives better results for better channel quality conditions.

\section{CONCLUSION}

In this paper, we proposed a new RNTI aggregation method for enhancing control capacity for a Multi-users Multiplexing Radio Voice Transmission method. This method uses PDCCH channel with high format, created by the aggregation of PDCCH channels with low format, to transmit several RNTIs of different UEs in a same multiplexing group. The number of RNTIs transmitted in one PDCCH is calculated to ensure that there is no increase of BER for receiving PDCCH channel. The results show that the control capacity of the multiplexing scheme can rise up to $170 \%$.
The use of RNTI aggregation method for the Multi-users Multiplexing Radio Voice Transmission method allows reducing both data overhead and control overhead issues for VoLTE in PMR context. This ensures an augmentation of voice capacity for VoLTE in PMR context. However, an evaluation of the energy consumption at the receiver side needs to be considered because in this method, there is an increase in the size of the search space.

\section{REFERENCES}

[1] MC Nguyen, H Nguyen, A Florea, P Mege, L Martinot, and E Georgeaux. Architecture for Multi-users Multiplexing Radio Voice Transmission for Enhancing Voice Capacity over LTE in PMR Context. Personal, Indoor and Mobile Radio Communications (PIMRC), 2014.

[2] Xavier Pons Masbernat, Christophe Gruet, Frédéric Fraysse, Serge Contal, and Lirida Naviner. Green solutions for future lte pmr networks. In Computer Aided Modeling and Design of Communication Links and Networks (CAMAD), 2012 IEEE 17th International Workshop on, pages 165-167. IEEE, 2012.

[3] AA Florea, L Martinod, P Mege, and H Nguyen. Multi-layer Realistic Voice Capacity Evaluation in LTE Rel. 9 and Performance Comparison with PMR and GSM. In World Telecommunications Congress (WTC), 2012.

[4] C Bormann, C Burmeister, M Degermark, H Fukushima, H Hannu, LE Jonsson, R Hakenberg, T Koren, K Le, Z Liu, et al. RObust Header Compression (ROHC): Framework and four profiles: RTP, UDP, ESP, and uncompressed. Technical report, RFC 3095, July, 2001.

[5] ER Vale, MA Grivet, and JC Brandao. Reducing the VoIP packet overhead in UMTS air interface. In Adaptive Science \& Technology (ICAST), 2009.

[6] AA Florea, H Nguyen, L Martinod, and C Molko. Serial Progressive Hierarchical Turbo Codes. In Global Telecommunications Conference (GLOBECOM), 2011.

[7] Telesystem Innovations Inc. LTE in a Nutshell: The Physical Layer. 2010.

[8] D Jiang, H Wang, E Malkamaki, and E Tuomaala. Principle and performance of semi-persistent scheduling for VoIP in LTE system. In Wireless Communications, Networking and Mobile Computing (WiCom), 2007.

[9] Sean McBeath, Jack Smith, Doug Reed, Hao Bi, Danny Pinckley, Alfonso Rodriguez-Herrera, and Jim O'Connor. Efficient signaling for VoIP in OFDMA. In Wireless Communications and Networking Conference (WCNC), 2007.

[10] R Susitaival and M Meyer. LTE coverage improvement by TTI bundling. In Vehicular Technology Conference (VTC), 2009.

[11] J Puttonen, T Henttonen, N Kolehmainen, K Aschan, M Moisio, and $\mathrm{P}$ Kela. Voice-over-IP performance in UTRA Long Term Evolution downlink. In Vehicular Technology Conference (VTC), 2008.

[12] Tayal Nitin. All about pdcch and cce allocation. http://www.test.org/ doe/.

[13] Evolved Universal Terrestrial Radio Access (E-UTRA); Physical layer procedures, 3GPP TS. 36.213 V9.0.1, Dec. 2009.

[14] Patrick Hosein. Resource allocation for the lte physical downlink control channel. In GLOBECOM Workshops, 2009 IEEE, pages 1-5. IEEE, 2009. 